\title{
Robotically Assisted CBCT-Guided Needle Insertions: Preliminary Results in a Phantom Model
}

\section{Antoine Pfeil, Roberto Luigi Cazzato, Laurent Barbé, Pierre De Marini, Jeanie}

Betsy Chiang, Julien Garnon, Pierre Renaud, et al.

CardioVascular and Interventional Radiology

ISSN 0174-1551

Cardiovasc Intervent Radiol DOI 10.1007/s00270-018-2088-8

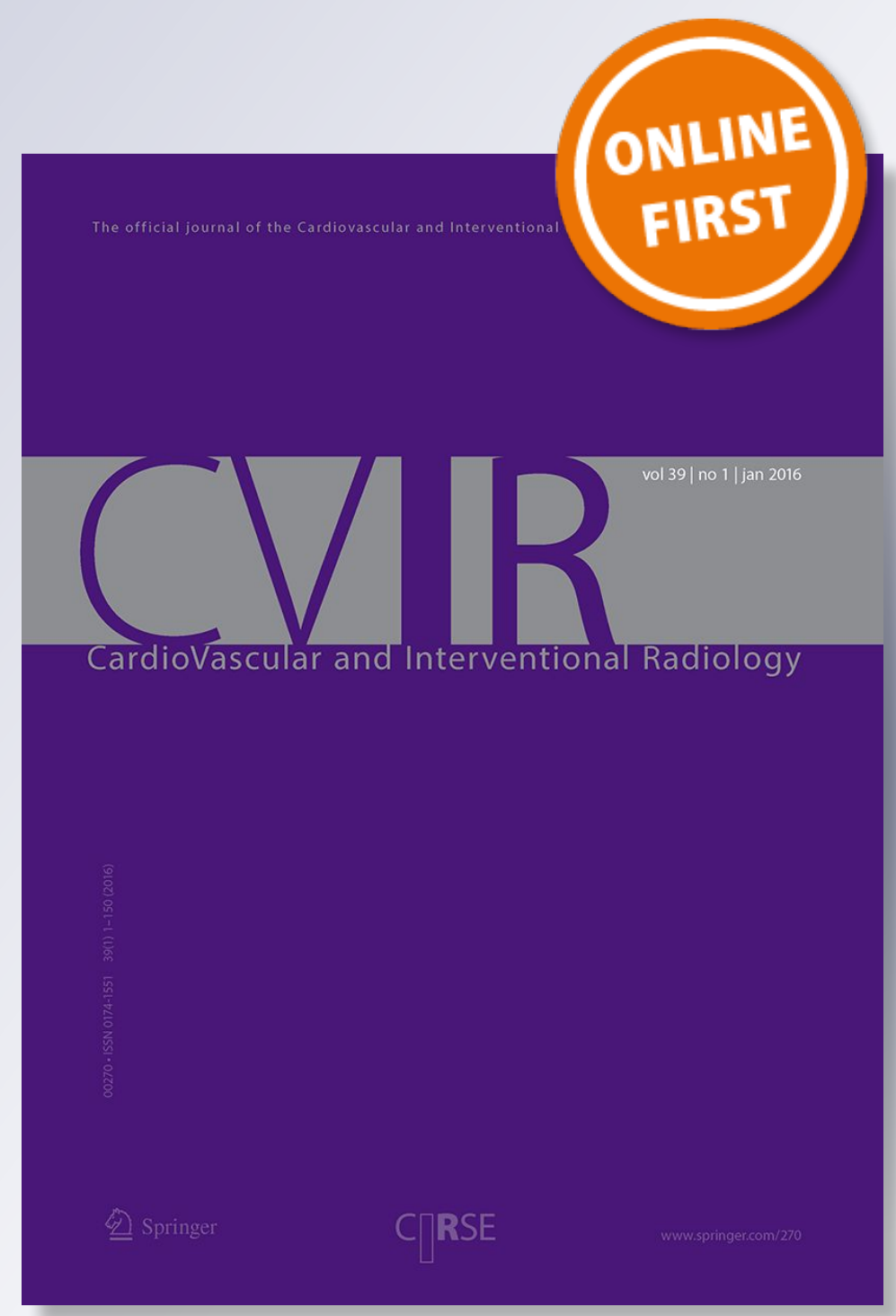

脰 Springer 
Your article is protected by copyright and all rights are held exclusively by Springer Science+Business Media, LLC, part of Springer Nature and the Cardiovascular and Interventional Radiological Society of Europe (CIRSE). This e-offprint is for personal use only and shall not be self-archived in electronic repositories. If you wish to selfarchive your article, please use the accepted manuscript version for posting on your own website. You may further deposit the accepted manuscript version in any repository, provided it is only made publicly available 12 months after official publication or later and provided acknowledgement is given to the original source of publication and a link is inserted to the published article on Springer's website. The link must be accompanied by the following text: "The final publication is available at link.springer.com". 


\title{
Robotically Assisted CBCT-Guided Needle Insertions: Preliminary Results in a Phantom Model
}

\author{
Antoine Pfeil ${ }^{1}$ - Roberto Luigi Cazzato ${ }^{2}$ Laurent Barbé ${ }^{1}$ Pierre De Marini ${ }^{2}$. \\ Jeanie Betsy Chiang ${ }^{3} \cdot$ Julien Garnon $^{2} \cdot$ Pierre Renaud $^{1} \cdot$ Afshin Gangi $^{2}$
}

Received: 14 July 2018/Accepted: 1 October 2018

(C) Springer Science+Business Media, LLC, part of Springer Nature and the Cardiovascular and Interventional Radiological Society of Europe (CIRSE) 2018

\begin{abstract}
Aim To compare robotic-assisted needle insertions performed under CBCT guidance to standard manual needle insertions.

Materials and Methods A homemade robotic prototype was used by two operators to perform robotic and manual needle insertions on a custom-made phantom. Both the operators had no experience with the prototype before starting the trial. The primary endpoint was accuracy (i.e., the minimal distance between the needle tip and the center of the target) between robotic and manual insertions. Secondary endpoints included total procedure time and operators' radiation exposure. The Wilcoxon test was used. A $p$ value less than 0.05 was considered statistically significant.

Results Thirty-three (17 manual, 16 robotic) needle insertions were performed. Mean accuracy for robotic
\end{abstract}

Antoine Pfeil and Roberto Luigi Cazzato have contributed equally to this work.

Antoine Pfeil

a.pfeil@unistra.fr

Roberto Luigi Cazzato

gigicazzato@ hotmail.it

Laurent Barbé

laurent.barbe@unistra.fr

Pierre De Marini

pierre.demarini@gmail.com

Jeanie Betsy Chiang

jbchian@gmail.com

Julien Garnon

julien.garnon@chru-strasbourg.fr

Pierre Renaud

pierre.renaud@unistra.fr insertion was $2.3 \pm 0.9 \mathrm{~mm}$ (median 2.1; range 0.8-4.2) versus $2.3 \pm 1 \mathrm{~mm}$ (median 2.1; range 0.7-4.4) for manual insertion $(p=0.84)$. Mean procedure time was $683 \pm 57 \mathrm{~s}$ (median 670; range 611-849) for the robotic group versus $552 \pm 40 \mathrm{~s}$ (median 548; range 486-621) for the manual group $(p=0.0002)$. Mean radiation exposure was 3.25 times less for the robotic insertion on comparison to manual insertion for the operator $1(0.4 \mathrm{vs} 1.3 \mu \mathrm{Gy})$; and 4.15 times less for the operator 2 (1.9 vs $7.9 \mu \mathrm{Gy})$.

Conclusion The tested robotic prototype showed accuracy comparable to that achieved with manual punctures coupled to a significant reduction of operators' radiation exposure. Further, in vivo studies are necessary to confirm the efficiency of the system.

Keywords Percutaneous · biopsy · Cone-beam CT · Robot $\cdot$ Radiation dose
Afshin Gangi
gangi@unistra.fr
1 ICube Laboratory, University of Strasbourg, CNRS, INSA Strasbourg, 1, place de l'Hôpital, 67091 Strasbourg Cedex, France
2 Department of Interventional Radiology, University Hospital of Strasbourg, 1, place de l'Hôpital, 67091 Strasbourg Cedex, France
3 Interventional Radiology, Queen Marie Hospital, Hong Kong, 102 Pok Fu Lam Rd, Pok Fu Lam, Hong Kong 


\section{Introduction}

In the modern era of medicine with increased need for tailored therapy [1], the demand for interventional radiologists performing percutaneous image-guided biopsy has rapidly increased [2]. Although ultrasound and computed tomography (CT) remain the most commonly used guidance techniques, an expansion of other modalities has been observed [2-4]. In this regard, flat-panel cone-beam CT (CBCT) has been a rapidly emerging modality. It combines real-time fluoroscopy and CT-like imaging designed to be used for interventional purposes [5-7]. In addition, the development of navigation software that accurately facilitates needle tracking and deployment has contributed to the popularity of these machines. However, despite all the aforementioned advantages, CBCT does have its shortcomings, such as exposing operators to radiation. Moreover, successful tissue sampling largely depends on precise targeting thus requiring experienced operators, which is not always available in all institutions. Accordingly, assisting the human with robotic systems has been proposed; and experimental tests have been performed mainly on phantom models, to highlight the advantages and drawbacks of robotic systems [8, 9]. Schulz et al. [8] proved the feasibility and accuracy of a commercially available robotic system on a phantom model under CBCT guidance. However, no comparison with standard manual punctures and no operators' radiation exposure was reported; moreover, the used device did not allow remote needle insertion. Hiraki et al. [9] tested a floor-mounted robot with remote needle insertion and demonstrated that the system was accurate when used in phantoms (as compared to manual punctures) and animals, without radiation exposure to the physician. However, their device was specially designed for CT scanners provided with a sliding gantry, thus not being of easy use with all CT machines.

In this study, a prototype robot allowing remote needle control during CBCT-guided needle insertions was tested on a phantom to evaluate the accuracy, operator's radiation exposure and procedure time as compared to standard manual needle insertions.

\section{Materials and Methods}

No local Institution Review Board approval was needed for this study.

\section{Robotic System}

The robot is a light $(250 \mathrm{~g})$ homemade prototype composed of a slave device (110 mm high, $250 \mathrm{~mm}$ long) and a master interface (Fig. 1). The slave component consists of three modules: the first allows manual installation of the robot on the phantom by four suction cups; the second allows for needle orientation; and the third for needle insertion.

The needle orientation module permits two degrees of freedom by the means of two rotational joints. Each joint is remotely controlled by a pair of antagonistic cables woundup around a pulley on the master interface. The operator can adjust the angle of the joints by manually turning two knobs on the master interface. Needle orientation can vary $\pm 40^{\circ}$ in the cranio-caudal plane and $\pm 36^{\circ}$ in the transverse plane regardless of the entry point.

The insertion module consists of a pneumatic actuator where the needle is held; it generates force up to $10 \mathrm{~N}$, allowing for needle progression at a speed of $1.4 \mathrm{~mm} / \mathrm{s}$. Operators activate needle insertion through a foot pedal.

\section{Needle Insertions}

Two operators with 3 and 5 years of experience, respectively, in percutaneous image-guided biopsy performed a series of robotic and manual needle insertions on a custommade phantom $(60 \mathrm{~mm} \times 250 \mathrm{~mm} \times 140 \mathrm{~mm})$, which was made of a superficial sheet of silicone and an inner layer of soft polyvinyl chloride (Bricoleurre, Mont Saint Aignan, France). The surface of the phantom mimics the elastic properties of human skin, and the inner part mimics that of human liver [10]. The phantom contained 40 radiopaque spherical targets (diameter $6 \mathrm{~mm}$ ). Each target was identified by a number. All targets were located $50 \mathrm{~mm}$ deep from the surface and were placed in the phantom with the intention of being approached with a double-angulated puncture (i.e., the operator was obliged to move the robotic arm on both cranio-caudal and axial directions in order to reach the selected target). As a result, in all cases, the targets were approached with a $60-\mathrm{mm}$ needle pathway, which was angulated $33.5^{\circ}$ with respect to the vertical axis of the phantom. Given the fixed distance of the targets from the surface of the phantom, and the need for a double-angulated approach, it could be reasonably estimated that all punctures were equivalent in terms of technical dexterity.

Both the operators were blinded to the targets' location before starting the trial. For each insertion, the operator randomly selected a sealed envelope containing the number corresponding to the assigned target to be punctured either manually or with robotic assistance.

All the insertions were performed under CBCT guidance (Allura FD20, Philips Healthcare, the Netherlands). The CBCT unit was provided of software for needle planning and deployment (XperCT and XperGuide, Philips Healthcare). Applied parameters of image acquisition were: 

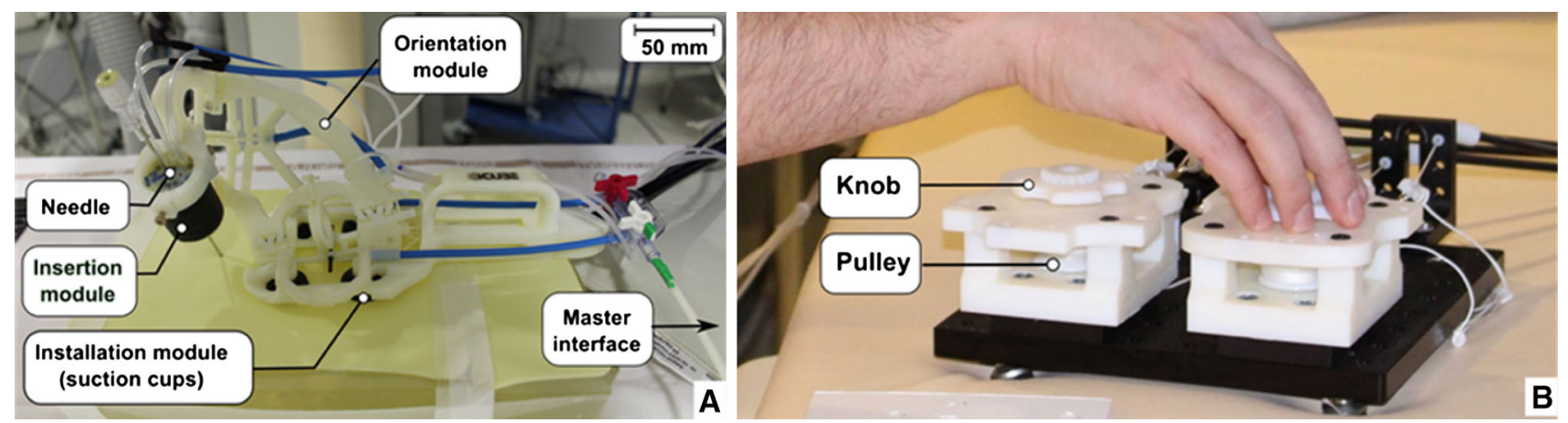

Fig. 1 The robotic prototype with all its different components: the slave device (A); the master interface (B)

source-detector distance $1200 \mathrm{~mm}$; rotation speed $18 \%$; rotation duration $10 \mathrm{~s}, 30$ images/s; total arc trajectory range 180; number of projections 300 images; matrix size $1024 \times 1024$; and automatic exposure control.

During all needle insertions, operators wore plastic gloves containing eight thermo-luminescent dosimeters (TLD dosimeter, type GR200A, Fimel, France) to measure the radiation exposure. Each operator wore different pair of gloves for robotic and manual insertion, allowing interpretation of the difference in radiation exposure between the two techniques. In both manual and robotic insertion, a $15 \mathrm{G}$ needle was used.

\section{Robotic Insertions}

None of the operators had experience with the robot before starting the trial. Each operator was allowed a 10-min initial practice with the robot to familiarize with the workings of the robotic prototype before starting the trial; during this phase, no needle insertions were allowed. Operators were instructed to perform robotic insertions first followed by manual insertions.

The procedure started with a CBCT imaging acquisition, and the acquired frames were transferred on a dedicated workstation (Xtravision R8.8, Philips Healthcare). Thereafter, the operator randomly selected the envelope indicating the assigned target, which was subsequently identified on the acquired images; needle trajectory using the XperGuide software was subsequently planned, and a fusion of the fluoroscopic image and the CBCT slice with the trajectory (displayed either with a "bulls' eye" view or a "sagittal view") was created and used to deploy the needle. The operator manually positioned the robot holding the needle at the planned entry point under fluoroscopic guidance and fixed it on the phantom by activating the suction cups. The needle was then remotely orientated to align with the fused CBCT image containing the needle trajectory on the "bulls' eye" view.
After a small incision on the surface of the phantom, the needle was advanced by activating the pneumatic actuator until the needle reached the target on the "sagittal view" (Fig. 2). During the first $2 \mathrm{~cm}$ of needle insertion, if needed, operators were allowed to adjust the needle orientation in both directions. At any time during targeting (including robot installation on the phantom) and needle deployment, operators were allowed to perform real-time fluoroscopy as per their usual.

A final 3D CBCT acquisition was performed to show the final position of the needle tip in relation to the target.

\section{Manual Insertions}

Manual insertions followed the same workflow as robotic insertions. The operator started with the "bulls' eye" view to target the assigned entry point by handling the needle with a surgical forceps. After a small incision on the surface of the phantom, the needle was advanced by operators by using the "bulls' eye" and the "sagittal" views. As it happened for robotic insertions, operators were allowed to perform real-time fluoroscopy during needle deployment as per their usual. A final 3D CBCT acquisition was performed to show the final position of the needle tip in relation to the target.

\section{Data Collection and Analysis}

For each insertion, accuracy and total procedure time were collected. Accuracy was defined as the minimal distance between the needle tip and the center of the target according to the latest multi-planar 3D CBCT images. The 3D distance was measured directly on the imaging software (XperCT, Philips Healthcare, the Netherlands) at the end of each puncture in consensus by one author (non-performing the puncture) and one technician.

The number of required needle insertions was calculated to determine $1 \mathrm{~mm}$ of equivalence in accuracy between the robotic and the manual insertions. Absolute mean 


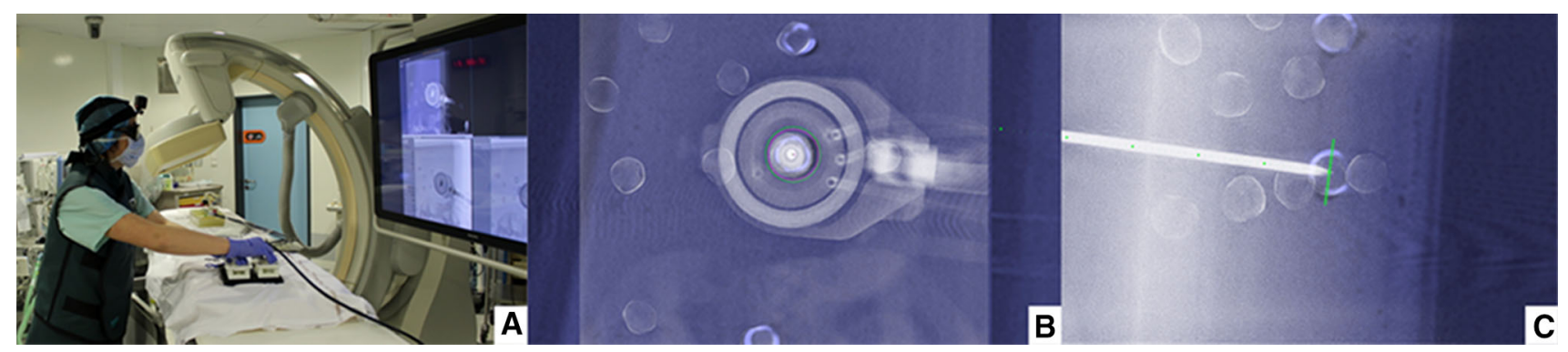

Fig. 2 Robotic insertion. Once the robot was manually positioned and fixed on the phantom, the operator remotely orientated it $(\mathbf{A})$ in order to align with the fused CBCT image containing the needle trajectory on the "bulls' eye" view (B); after a small incision on the

difference of accuracy between both groups was estimated to be $1 \mathrm{~mm}$ (with $1 \mathrm{~mm}$ standard deviation for both groups). With $\alpha$ value of 0.05 and $\beta$ value of 0.2 , at least 16 insertions were required for each group.

Total procedure time included the time needed to acquire the 3D dataset for trajectory planning, orientation and deployment of needle, as well as the time needed to acquire CBCT images to check accuracy.

Operator's radiation exposure for both series of insertions (manual vs robotic) was measured on the operators' hands. In particular, it was calculated by subtracting the background dose registered by a reference TLD dosimeter placed in the control room from the highest final cumulative dose registered by any of the TLD dosimeters embedded on one of the two gloves worn by the operator.

The primary endpoint was accuracy between manual and robotic punctures; secondary endpoints included total procedure time and operators' radiation exposure.

The Wilcoxon test was used to compare all the tested variables. A $p$ value less than 0.05 was considered statistically significant.

\section{Results}

Thirty-three (17 manual, 16 robotic) needle insertions were performed. During robotic insertions, needle orientation was adjusted along the first $2 \mathrm{~cm}$ of the planned trajectory in $12 / 16(75 \%)$ cases. Mean accuracy for robotic insertion was $2.3 \pm 0.9 \mathrm{~mm}$ (median 2.1; range 0.8-4.2) vs $2.3 \pm 1 \mathrm{~mm}$ (median 2.1; range 0.7-4.4) for manual insertion ( $p=0.84$; Fig. 3$)$. Mean procedure time was $683 \pm 57$ s (median 670; range 611-849) for the robotic group vs $552 \pm 40 \mathrm{~s}$ (median 548; range 486-621) for the manual group $(p=0.0002)$. There was no significant difference in accuracy and procedure time between both operators in either manual or robotic insertions (Table 1).

Mean radiation exposure per insertion was 3.25 times less for the robotic insertions on comparison to manual surface of the phantom, the needle was advanced by activating the pneumatic actuator until the needle reached the target on the "sagittal view" (C)

ones for the operator $1(0.4$ vs $1.3 \mu \mathrm{Gy})$; and 4.15 times less for the operator 2 (1.9 vs $7.9 \mu \mathrm{Gy})$.

\section{Discussion}

Several different robotic systems have been developed and are commercially available to facilitate percutaneous targeting and needle deployment [11]; most of these systems have been designed for CT guidance with a reported accuracy $\leq 1.5 \mathrm{~mm}$. In the present study, a robotic prototype was tested under CBCT guidance on a homemade phantom. Assuming a tolerated $1 \mathrm{~mm}$ difference in accuracy between robotic and manual insertions, similar accuracies were noted in both groups by both operators in all the different punctures performed. Results also indicated that the operators were equivalent in terms of experience, as demonstrated by the comparable accuracy in manual insertions. On average, the robot demonstrated accuracy of $2.3 \pm 0.9 \mathrm{~mm}$, which is within the accepted range if one

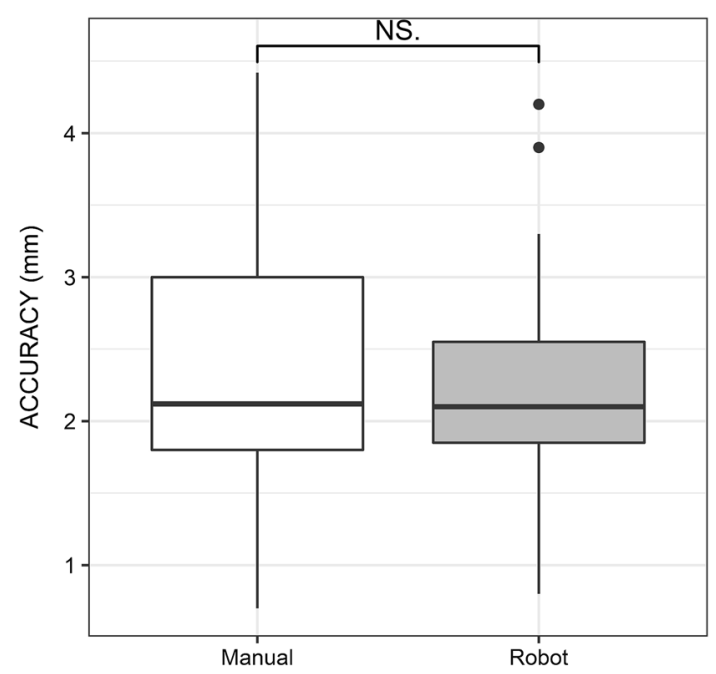

Fig. 3 Overall accuracy in the study (NS $=p$ statistically not significant) 
Table 1 Tested variables according to different operators

\begin{tabular}{|c|c|c|c|}
\hline & Operator 1 & Operator 2 & $p$ \\
\hline \multicolumn{4}{|l|}{ Manual } \\
\hline N. Insertions & 8 & 9 & \\
\hline \multicolumn{4}{|l|}{ Accuracy $(\mathrm{mm})$} \\
\hline Mean \pm SD & $2.2 \pm 1.1(0.7-4.4)$ & $2.4 \pm 1(0.7-3.4)$ & 0.44 \\
\hline Median & 2.1 & 2.8 & \\
\hline \multicolumn{4}{|l|}{ Total procedure time $(\mathrm{s})$} \\
\hline Mean \pm St. Dev. & $536 \pm 31(496-596)$ & $566 \pm 45(486-621)$ & 0.14 \\
\hline Median & 534 & 571 & \\
\hline Total cumulative dose $(\mu \mathrm{Gy})$ & 10.55 & 71.3 & \\
\hline \multicolumn{4}{|l|}{ Robotic } \\
\hline N. Insertions & 7 & 9 & \\
\hline \multicolumn{4}{|l|}{ Accuracy $(\mathrm{mm})$} \\
\hline Mean \pm St. Dev. & $2.6 \pm 0.6(1.9-3.9)$ & $2.1 \pm 1(0.8-4.2)$ & 0.09 \\
\hline Median & 2.4 & 1.9 & \\
\hline \multicolumn{4}{|l|}{ Total procedure time $(\mathrm{s})$} \\
\hline Mean \pm St. Dev. & $669 \pm 50(611-748)$ & $694 \pm 63(633-849)$ & 0.46 \\
\hline Median & 663 & 671 & \\
\hline Total cumulative dose $(\mu \mathrm{Gy})$ & 2.61 & 16.8 & \\
\hline
\end{tabular}

assumes that most of the common biopsy targets measure $\geq 10 \mathrm{~mm}$ [11, 12]. Despite differing operator habits and the fact that radiation dose was only estimated by one of the several embedded TLD dosimeters, the radiation dose to operators' hands was significantly reduced with the robotic insertions. This result was largely expected since needle orientation and deployment with the robot were performed remotely. Such approach represents one of the main advantages granted by robotic insertion [9], which allows the operator to monitor needle advancement through fluoroscopy without receiving significant radiation exposure.

In terms of procedure time, robotic-assisted insertions required on average 2 min more than manual insertions merely because manual advancement of the needle was faster than the capped actuator speed of $1.4 \mathrm{~mm} / \mathrm{sec}$ (which can be increased). Nevertheless, overall procedure time with the robot was still acceptable particularly considering that this was the first time the operators used the device, thus suggesting a short learning time.

The presented prototype is similar to the iSYS system (Medizintechnik GmbH, Kitzbuehel, Austria), which was tested in phantom, animal and human models under CBCT or CT guidance $[8,12]$. Compared to the iSYS, the prototype used in the present study has the advantage to be attached directly to the phantom rather than to the CT or CBCT table, which allows the operator to work in a condition more similar to the classic manual puncture. Schulz et al. [8] performed 40 iSYS-assisted needle insertions on a custom-made phantom under CBCT guidance. Navigation software was used to assist the trajectory planning and needle deployment. They did not measure the operators' radiation exposure nor did they compare to manual insertion. However, they noted an overall accuracy of $1.1 \mathrm{~mm}$; such gap was further reduced to $0.3 \mathrm{~mm}$ when the orientation process was done with the needle in place in the dedicated holder. It is probable that the discrepancy of accuracy between their study and the present study is due to the different materials used to construct the phantom, as Schulz et al. used simple sponge which was more easy to be penetrated by the needle compared to polyvinyl used in this study. In fact, especially in the series of punctures performed by the second operator who intensively used the fluoroscopy to follow needle advancement, a certain degree of elastic recoil was noted while the needle approached the target. This phenomenon was also described by Kettenbach et al. [12] who used a polymeric phantom to test the iSYS system under CT guidance. Interestingly, their accuracy (2.3 $\pm 0.8 \mathrm{~mm}$; range $0.9-3.7)$ was very similar to that noted in the present experience. Lastly, Groetz et al. [13] successfully tested the iSYS system on an ex vivo swine spinal model and in three humans undergoing spine biopsies. Nevertheless, clinical experience with robotic devices remains limited at the moment; and one of the most probable reasons accounts for the difficulty of robotic systems to compensate for target movements such as physiological breathing. Although some solutions such as a stepwise check approach with progressive corrective angulations along the needle pathway [14] have been proposed, concerns still remain as shown by the fact that most of the experience gained with robots is confined to phantoms and animal tests; and when human applications 
started, relative immobile targets such as the spine [13], or relatively large targets $(\geq 20 \mathrm{~mm}$ ) [15] were chosen.

The main limitation of the present study is related to the lack of in vivo tests, thus not allowing estimation of accuracy with moving targets, located at different depths from the surface. Moreover, the phantom model underestimates the clinical scenario, where the needle will be required to pass through tissues with different elastic properties and different thicknesses according to the patients' morphotype. Furthermore, a $15 \mathrm{G}$ needle was used, which is expected to be more easily directed and less subjected to deflection as compared the $18-20 \mathrm{G}$ needles that are more commonly used in clinical practice. Nevertheless, this preliminary experience was intended to validate the prototype before further implementations, development and tests. Lastly, despite the potential application of the prototype under CT or MRI guidance, further evaluation of these modalities will have to be tackled in the next future.

In conclusion, the tested robotic prototype showed accuracy comparable to that achieved with manual punctures under CBCT guidance in a phantom model; moreover, the robot allowed for a significant reduction of operators' radiation exposure. Further, in vivo studies are necessary to confirm the efficiency of the system.

Acknowledgements Authors would like to sincerely thank Marie Charlotte Hesler (MD) and Emile Reeb for their participation in the experiments; Luc Mertz (Ph.D.), Nicolas Clauss and Elise Bottlaender from the Radiophysics and Radioprotection Department of the University of Strasbourg for their kind assistance in lending and reading the TLD dosimeters.

Funding This work was supported by the INTERREG Upper Rhine program from the ERDF (European Regional Development Fund), SPIRITS project.

\section{Compliance with Ethical Standards}

Conflict of interest The authors declare that they have no conflict of interest.

Human and Animal Rights This article does not contain any studies with animals performed by any of the authors.

\section{References}

1. Tsai EB, Pomykala K, Ruchalski K, et al. Feasibility and safety of intrathoracic biopsy and repeat biopsy for evaluation of programmed cell death ligand-1 expression for immunotherapy in non-small cell lung cancer. Radiology. 2018;287(1):326-32.

2. Veltri A, Bargellini I, Giorgi L, et al. CIRSE guidelines on percutaneous needle biopsy (PNB). Cardiovasc Intervent Radiol. 2017;40(10):1501-13.

3. Cazzato RL, Garnon J, Shaygi B, et al. PET/CT-guided interventions: indications, advantages, disadvantages and the state of the art. Minim Invasive Ther Allied Technol. 2018;27(1):27-32.

4. Garnon J, Koch G, Tsoumakidou G, et al. Ultrasound-guided biopsies of bone lesions without cortical disruption using fusion imaging and needle tracking: proof of concept. Cardiovasc Intervent Radiol. 2017;40(8):1267-73.

5. Lee SM, Park CM, Lee KH, et al. C-arm cone-beam CT-guided percutaneous transthoracic needle biopsy of lung nodules: clinical experience in 1108 patients. Radiology. 2014;271(1):291-300.

6. Braak SJ, van Melick HHE, Onaca MG, et al. 3D cone-beam CT guidance, a novel technique in renal biopsy-results in 41 patients with suspected renal masses. Eur Radiol. 2012;22(11):2547-52.

7. Cazzato RL, Battistuzzi J-B, Catena V, et al. Cone-beam computed tomography (CBCT) versus $\mathrm{CT}$ in lung ablation procedure: which is Faster? Cardiovasc Intervent Radiol. 2015;38(5):1231-6.

8. Schulz B, Eichler K, Siebenhandl P, et al. Accuracy and speed of robotic assisted needle interventions using a modern cone beam computed tomography intervention suite: a phantom study. Eur Radiol. 2013;23(1):198-204.

9. Hiraki T, Kamegawa T, Matsuno T, et al. Robotically driven CTguided needle insertion: preliminary results in phantom and animal experiments. Radiology. 2017;285(2):454-61.

10. Lefebvre PM, Koon KTV, Brusseau E, et al. Comparison of viscoelastic property characterization of plastisol phantoms with magnetic resonance elastography and high-frequency rheometry. In: 38th Annual International Conference of the IEEE Engineering in Medicine and Biology Society (EMBC). 2016. pp. 1216-9.

11. Kettenbach J, Kronreif G. Robotic systems for percutaneous needle-guided interventions. Minim Invasive Ther Allied Technol. $2015 ; 24(1): 45-53$.

12. Kettenbach J, Kara L, Toporek G, et al. A robotic needle-positioning and guidance system for CT-guided puncture: ex vivo results. Minim Invasive Ther Allied Technol. 2014;23(5):271-8.

13. Groetz S, Wilhelm K, Willinek W, et al. A new robotic assistance system for percutaneous CT-guided punctures: initial experience. Minim Invasive Ther Allied Technol. 2016;25(2):79-85.

14. Ben-David E, Shochat M, Roth I, et al. Evaluation of a CTguided robotic system for precise percutaneous needle insertion. J Vasc Interv Radiol. 2018. https://doi.org/10.1016/j.jvir.2018.01. 002.

15. Solomon SB, Patriciu A, Bohlman ME, et al. Robotically driven interventions: a method of using CT fluoroscopy without radiation exposure to the physician. Radiology. 2002;225(1):277-82. 